\title{
AVALIAÇAO DA CONDIÇÃO NUTRICIONAL DAS GESTANTES NO MUNICÍPIO DE SANTO ANDRE, UTILIZANDO O GRAFICO DE ROSSO'
}

\section{ASSESSING THE NUTRITIONAL CONDITION OF PREGNANT WOMEN IN THE CITY OF SANTO ANDRÉ THROUGH ROSSO'S GRAPH}

Roseli S. Sarni ${ }^{1}$

Denise Scloeps ${ }^{2}$

Cristiane Kochi ${ }^{2}$

Camila V. Mathias ${ }^{3}$

Cláudia R. P. Oliveira ${ }^{3}$

Isabela M. de Vuono 3

Melissa V. Bueno ${ }^{3}$

Nilton G. Santos Junior ${ }^{1}$

Sérgio R. Longo ${ }^{1}$

Dráuzio Viegas ${ }^{4}$

SARNI, R.S.; SCHOEPS, D.; KOCHI, C.; MATHIAS, C.V.; OLIVEIRA, C.R.R; VUONO, I.M.; BUENO, M.V.; SANTOS JUNIOR, N.G.; LONGO, S.R.; VIEGAS, D. Avaliação da condição nutiicional das gestantes no Municipio de Santo André, utilizando o gráfico de Rosso. Rev. Bras. Cresc. Desenv. Hum., São Paulo, 9(1),1999.

Resumo: A falta de vigilancia da condição nutricional da gestante durante o exame pré-natal tem dificultado intervenções adequadas no sentido de prevenir a ocorrência do baixo peso ao nascimento (BP). Foram avaliadas, prospectivamente, 95 gestantes de baixo nível sócio-econômico do Município de Santo André, para realização de exame pré-natal, utilizando-se a curva de Rosso para sua classificação nutricional. O percentual de mãe com baixo peso foi de $36,8 \%$. As mães com mais de duas gestações apresentaram maior ocorrência de sobrepeso ou obesidade e as com menos de duas gestações, percentual significativamente maior de baixo peso. A anemia também se associou de maneira significativa às gestantes de baixo peso. $\mathrm{O}$ presente trabalho ressalta a importancia da implantação de instrumento na rede básica de saúde para controlar o ganho de peso da gestante, bem como da vigilancia de outros fatores de risco no intuito de reduzir os elevados percentuais de recém-nascidos com BP.

Palavras-chave: gestante; baixo peso ao nascimento; pré-natal.

1 Professoras Assistentes da Disciplina de Pediatria e Puericultura do Departamento de Saúde Matcrno Infantil da Faculdade de Medicina da Fundação do ABC.

2 Médica Responsável pelo Serviço de Endocrinologia Pediátrica do Núcleo de Nutrição - Secretaria do Estado da Saúde - São Paulo.

3 Acadêmicos do $6^{\circ}$ ano da Faculdade de Medicina da Fundação do ABC.

4 Professor Titular da Disciplina de Pediatria e Puericultura do Departamento de Saúde Materno infantil da Faculdade de Medicina da Fundação do ABC.

- Trabalho realizado pela Disciplina de Pediatria e Puericultura da Faculdade de Medicina da Fundação do ABC - Santo André - SP

- Trabalho apresentado na XXXVI Reunião da Sociedade Latino Americana de Investigação Pediatrica, Xll Reunião da Sociedade Latino Americana de Endocrinologia Pediátrica, II Reunião da Associação Latino Americana de Nefrologia Pediátrica - São Pedro- SP- 1998. - Trabalho apresentado no XXIII Congresso Universitário da Faculdade de Medicina da Fundação do ABC - Santo Andre - SP 1998.

- Correspondência: Dra. Roseli S. Sarni - Endereço: Rua Renê Zamlutti, M - apto. s/2 - Vila Mariana - São Paulo - SP CEP: 04116-260 - E-mail: HYPERLINK mailto: sarni@originet.com.br 


\section{INTRODUÇÃO}

O baixo peso ao nascer é definido pela OMS como o peso de nascimento menor que 2.500 gramas. O peso ao nascimento é fator fundamental para sobrevivência e qualidade de vida da criança (PUFFER \& SERRANO, 1987), sendo considerado um dos melhores indicadores de saúde de uma população (PUFFER \& SERRANO, 1973).

$\mathrm{O}$ risco de se ter uma criança com baixo peso ao nascer é três vezes mais elevado nos países subdesenvolvidos que nos desenvolvidos. A taxa de mortalidade perinatal das crianças cujo peso ao nascer situa-se abaixo do $3^{\circ}$ percentil, pode ser até 6 vezes superior à de criança com peso adequado para a idade gestacional (ROCHA, 1991).

De acordo com a Pesquisa Nacional de Demografia em Saúde (PNDS, 1996), o baixo peso ao nascer ocorreu em 10,2\% das crianças brasileiras, valor este semelhante ao encontrado na década anterior. Este mesmo estudo, entretanto, revela um aumento no percentual de mães que realizaram exame pré-natal de 74\% (1986) para 85,2\% (1996) o que reforça o fato de que a falta de instrumentos para avaliar o estado nutricional de gestantes, em nosso meio, inviabiliza uma efetiva prevenção e conseqüente redução do número de recém-nascidos desnutridos já na vida intra uterina.

Vários fatores atuam na gestação repercutindo no peso do recém-nascido, dentre os quais, destacamos: drogas (fumo, álcool, cocaína, ópio e maconha); altitude elevada; estresse psicológico; processos infecciosos; defeitos congênitos; intervalo interpartal curto; multiparidade; extremos de idade; baixa estatura; cuidados pré-natais inadequados e má condição nutricional materna (PEREIRA, 1993).

Existem evidências de que um percentual relevante de mulheres brasileiras está em risco ou sofre de desnutrição. A baixa estatura demonstrada por uma parcela dessas mulheres sugere ter a desnutrição ocorrido durante a infância (TADDEI et al, 1991). Os recém-nascidos brasileiros apresentam menor média de peso, quando comparados aos de países desenvolvidos (PUFFER \& SERRANO, 1987).

O seguimento nutricional adequado da mulher que inicia a gestação desnutrida e a recuperação nutricional que se pode obter nesse período, manifestada por ganho de peso satisfatório, pode diminuir consideravelmente o risco de nascimento de criança com baixo peso (TADDEI et al, 1991). Em estudo multicêntrico (metanálise) coordenado pela OMS (KELLY; KEVANY; ONIS; SHAH, 1996) foi verificada forte correla- ção entre o baixo peso materno aferido com 1620 ou 24-28 semanas de gestação e o comprometimento ponderal do recém-nascido.

A "curva de ganho de peso da gestante" de Rosso (1985) permite a identifição da desnutrição materna já no segundo mes de gestação, bem como a ponderação de ganhos diferentes para gestantes de diversas estaturas, com base em critérios de adequação de peso/altura, para diferentes idades gestacionais. Esta curva foi padronizada pelo Ministério da Saúde do Brasil, em 1986, através de suas normas de assistência pré-natal (Brasil - Ministério da Saúde - Normas e Manuais Técnicos 36, 1998). Estudo realizado por PEREIRA (1993) com 21.125 gestantes, com idade gestacional entre $10^{\mathrm{a}}$ e $42^{\mathrm{a}}$ semanas de gestação durante a Campanha de Vacinação contra a Poliomielite em 22/09/90 que compareceram espontaneamente às Unidades Básicas de Saúde do Estado de São Paulo, revelou de acordo com a curva de Rosso:

- 28,3\% apresentaram peso baixo para a idade gestacional, $34,3 \%$ sobrepeso ou obesidade e apenas 37,4\% eram normais do ponto de vista antropométrico;

- em todas as regiões avaliadas, o percentual de gestantes com peso baixo para a idade gestacional foi maior no final da gestação que no início.

Quando comparadas com outros procedimentos diagnósticos, a curva de Rosso demonstra ser bom instrumento, principalmente por se caracterizar como tecnologia simples, de baixo custo, que pode ser executada por pessoa técnica de nível médio bem treinada e que não depende da utilização de equipamentos sofisticados (TADDEI et al, 1991).

Embora inúmeros trabalhos demonstrem a relação entre o ganho insuficiente de peso durante a gestação e o baixo peso ao nascer, pouco ou quase nada se sabe a respeito do estado nutricional e percentual de anemia das gestantes do $\mathrm{Mu}-$ nicípio de Santo André (São Paulo).

Desta forma, decidimos avaliar o estado nutricional, níveis de hemoglobina e hematócrito e outros fatores de risco para o baixo peso ao nascer das gestantes matriculadas no pré-natal em 7 unidades de saúde distribuídas pelo Município de Santo André.

\section{CASUÍSTICA E MÉTODO}

Foram avaliadas 95 gestantes de baixo nível sócio-econômico, entre a $10^{\mathrm{a}}$ e a $42^{\mathrm{a}}$ semanas de gestação, atendidas nas Unidades Básicas de Saúde da FAISA, distribuídas pelo Município de Santo André, para realização de pré-natal. Esta 
avaliação foi realizada no período de abril a junho de 1998.

Para classificação da gestante quanto ao ganho de peso, utilizou-se a "Curva de Rosso", que utiliza como indicador o percentual de peso/ altura, em relação ao padrão de referência, nas diversas idades gestacionais. A partir destes dados, e com a utilização de um "nomograma" classificou-se as gestantes em 4 categorias: A - baixo peso, B - peso normal, C - sobrepeso e D - obesa. Em nosso estudo, consideramos como categoria C a somatória de gestantes sobrepeso e obesas.

Foram coletados, também, alguns dados de anamnese, tais como: faixa etária, estado marital, escolaridade, número de gestações, ocorrência de baixo peso ao nascer em gestações anteriores, hipertensão arterial sistêmica, hábito de fumar e intervalo inter-partal. Outros dados relacionados ao baixo peso ao nascer, como pressão arterial e níveis de hemoglobina e hematócrito foram colhidos do prontuário da gestante, com base na última consulta pré-natal.

As gestantes foram informadas do estudo e assinaram o termo de consentimento pós-informação.

Para análise dos resultados foram utilizados testes paramétricos, levando-se em conta a natureza das distribuições das variáveis estudadas (SEGEL, 1975). Assim, utilizou-se o Teste do Qui-Quadrado ou Teste exato de Fisher. Foi estabelecido o limite de 0,05 ou $5 \%(a=5 \%)$ como nivel para rej eição da hipótese de nulidade, assinalando-se com um asterisco $\left({ }^{*}\right)$ os valores significantes.

\section{RESUL TADOS}

Os resultados estão expressos na forma de gráficos e tabelas.

\section{DISCUSSÃO}

O estado nutricional da gestante pode ser avaliado por via indireta através de inquéritos alimentares, avaliação das condições sócio-econômicas e por variáveis antropométricas (GODFREY \& ROBINSON, 1998). No presente trabalho, realizado com 95 gestantes regularmente matriculadas no pré-natal em Unidades Básicas de Saúde do Municíuípio de Santo André, encontramos: 36,8\% das gestantes com baixo peso para a idade gestacional, 32,6\% sobrepeso ou obesas e 30,5\% normais (Figura 1). Estes níveis encontrados foram superiores aos relatados por PEREIRA (1993) para as gestantes com baixo peso.
ROSSO (1985) ressalta que quando a ingestão alimentar de ratas é reduzida, durante o penodo gestacional, a limites críticos, o feto não é favorecido em relação à mãe. A divisão de nutrientes parece estar dirigida no sentido de favorecer os tecidos maternos. $\mathrm{O}$ autor acredita que $\mathrm{o}$ fato da natureza proteger mais a mãe do que o feto parece razoável do ponto de vista da sobrevivência nas espécies, pois um recém-nascido normal, proveniente de mãe severamente espoliada teria poucas chances de sobrevivência, se a lactação não fosse adequadamente iniciada.

Figura 1 - Distribuição das Gestantes Segundo Estado Nutricional

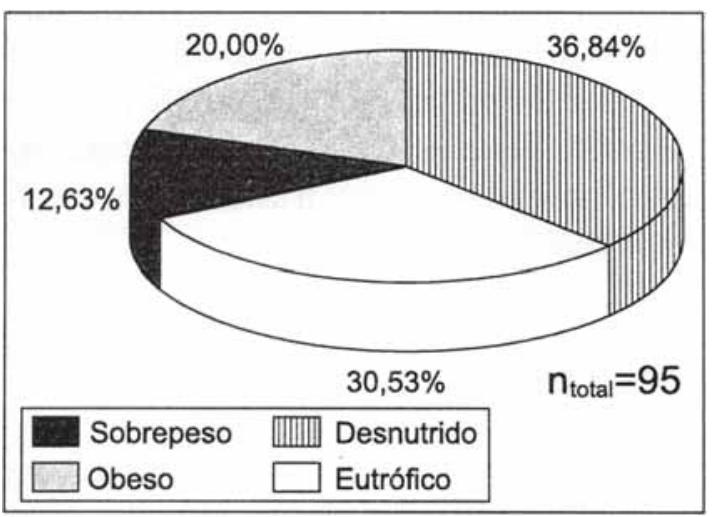

A vigilância dos agravos nutricionais de gestantes apresenta importante implicação prática, uma vez que em estudo recente realizado com 279 gestantes da Faculdade de Medicina de Botucatu (UNESP), as gestantes classificadas como de baixo peso pela curva de ROSSO apresentaram recém-nascidos com pesos mais baixos do que as mães normais, bem como maior proporção de recém-nascidos de termo pequenos para a idade gestacional e com menor espessura de prega cutânea tricipital (PINTO, 1996), justificando a necessidade ernergencial de implantação deste instrumento na rotina do exame pré-natal.

A falta de instrumentos para avaliar a evolução ponderal de gestantes durante a gravidez faz com que as intervenções não ocorram, mesmo em mães que realizam pré-natal, como as por nós avaliadas, impedindo-se a prevenção efetiva do baixo peso ao nascer.

Dentre outros fatores, o baixo peso ao nascer tem sido descrito como responsável pelos óbitos, principalmente os ocorridos no primeiro mes de vida, comprometendo ainda o crescimento e desenvolvimento daqueles que sobrevivem, tendo em vista sua dificuldade de adaptação ao meio extra-uterino, bem como as adversidades decorrentes das péssimas condições ambientais e só- 
cio-econômicas (LUCENA; LIMA \& MARINO, 1998).

O fumo exerce importante papel deletério como fornecedor de radicais livres de oxigênio (estima-se que cada tragada de fumo contenha 1017 radicais livres oxidantes) propiciando dano celular (CANTIN \& CRYSTAL, 1985) e necessidades nutricionais adicionais que acrescidas à demanda natural da gestação poderiam predispor a agravos do estado nutricional da gestante. Em nosso estudo, entretanto, não verificamos relação do hábito de fumar com baixo ganho de peso na gestação (Tabela l ).

Tabela 1 - Distribuição das gestantes segundo fator de risco Fumo versus Estado Nutricional

\begin{tabular}{c|r|c|c|c|c}
\hline \multirow{2}{*}{$\begin{array}{c}\text { Estado } \\
\text { Nutricional }\end{array}$} & \multicolumn{4}{|c|}{ Fumo } & \multirow{2}{*}{ Total } \\
\hline & \multicolumn{1}{|c|}{$\mathrm{n}$} & $\%$ & $\mathrm{n}$ & $\%$ & \\
\cline { 2 - 5 } $\mathrm{A}$ & 12 & 38,71 & 23 & 35,94 & 35 \\
$\mathrm{~B}$ & 7 & 22,58 & 22 & 34,38 & 29 \\
$\mathrm{C}$ & 12 & 38,71 & 19 & 29,69 & 31 \\
\hline Total & 31 & 100,00 & 64 & 100,00 & 95 \\
\hline \multicolumn{5}{|c}{ Teste do Qui-quadrado } \\
$\mathrm{AxB} \rightarrow \mathrm{x}^{2}=0,78 \quad$ AxBxC $\rightarrow \mathrm{x}^{2}=1,43$ \\
$\mathrm{BxC} \rightarrow \mathrm{x}^{2}=1,47$ & $\mathrm{x}^{2}$ Crítico $=5,99$ \\
$\mathrm{AxC} \rightarrow \mathrm{x}^{2}=0,14$ &
\end{tabular}

Figura 2 - Distribuição das Gestantes Segundo Faixa Etária

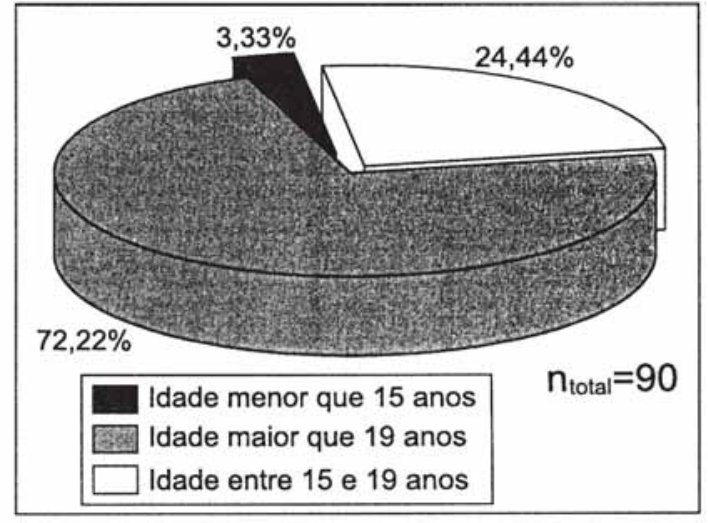

Outro fator importante e controverso na literatura diz respeito à idade materna. Encontramos um número elevado de adolescentes na amostra estudada (27,7\%) (Figura 2) reflexo, possivelmente do aumento da ocorrência de gestações nesta faixa etária. Estudo realizado por VITALLE (1994) com 6437 recém-nascidos e 109 óbitos fatais para avaliar a influência da idade materna (adolescência) sobre o produto da concepção (baixo nível sócio-econômico) não evidenciou alteração com respeito ao peso e comprimento do re- cém-nascido. Com base neste estudo, não realizamos a correlação entre idade materna e condição nutricional. Outro estudo realizado por MARIOTONI \& BARROS FILHO (1998), corroboraram os resultados anteriores, confirmando que a gravidez na adolescência não representou maior risco para a ocorrência de baixo peso ao nascer.

Outros indicadores relacionados em estudos anteriores à condição nutricional da gestantes foram avaliados: escolaridade materna (revelou que $51,5 \%$ das gestantes avaliadas eram analfabetas ou tinham o primeiro grau incompleto - Figura 3); estado marital (revelou que $16,84 \%$ das gestantes eram solteiras - Figura 4) e a ocorrência de baixo peso ao nascer nas gestações anteriores (14,3\% das gestantes: $n=49$ Figura 5).

Em nosso estudo, foi verificada diferença estatisticamente significante no número de anêmicas nas gestantes com baixo peso em relação às sobrepeso e obesas (Tabela 2). É fundamental a participação do ferro no perfeito crescimento do feto. É indiscutível o fato da gestação aumentar os requerimentos maternos desse oligoelemento, não só em função da demanda fetal, mas também para suprir a expansão da massa celular vermelha (SOUZA QUEIROZ; PEREIRA \& NÓBREGA, 1998). Em estudo retrospectivo realizado em 153.602 gestantes por SINISTERRARODRIGUEZ (1991), observou-se correlação significante entre os níveis de hemoglobina maternos inferiores a $10,5 \mathrm{mg} / \mathrm{dl}$ e a maior prevalência de baixo peso ao nascer e prematuridade. A associação de anemia e baixo peso na gestante aumenta ainda mais o risco de comprometimento no crescimento intra-uterino.

Com respeito à paridade encontramos um grande número de primigestas (46,3\%) e secundigestas $(22,1 \%)$ (Figura 6). Foi verificada influência estatisticamente significante do número de gestações na condição nutncional da gestante (Tabea 3). É descrito na literatura que o peso do recém-nascido aumenta a cada nova gravidez até a quinta gestação, a partir do que, começa a diminuir (SOUZA QUEIROZ et al, 1998). O curto período de amamentação também poderia explicar o maior número de gestantes com mais de duas gestações na categoria sobrepeso ou obesas.

As doenças infecciosas que podem acometer as gestantes elevam o gasto energético, podendo comprometer o ganho de peso gestacional. Neste trabalho foi avaliada, pela sua maior freqüencia, a infl uênia da ocorrência de infecção urinária no estado nutricional da gestante, não sendo observada diferença estatisticamente significante (Tabela 4). 
Figura 3 - Distnbuição das Gestantes Segundo Escolaridade

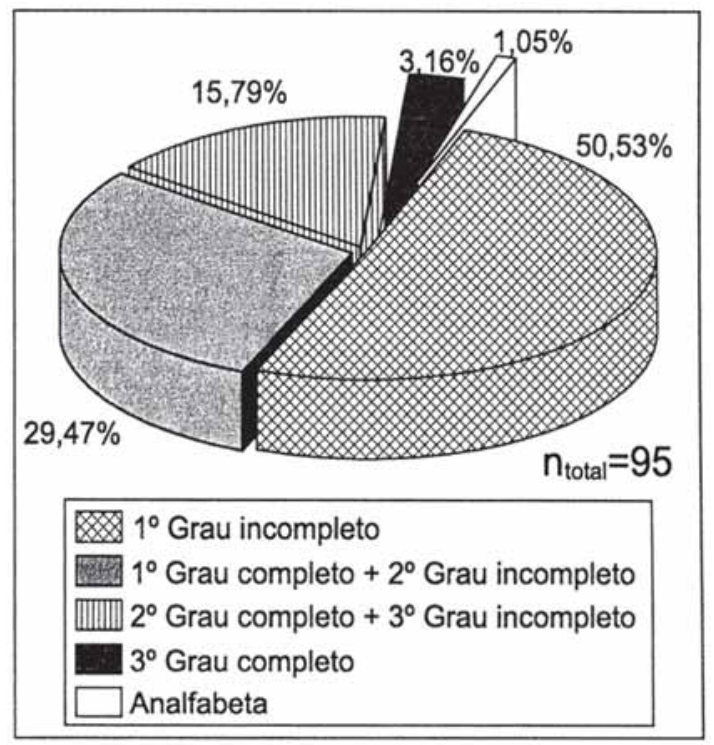

Figura 4 - Distribuição das Gestantes Segundo Estado Marital

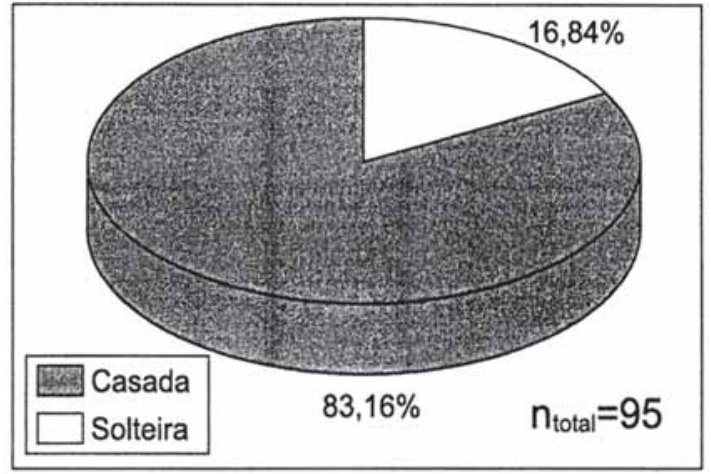

Figura 5 - Distribuição das Gestantes de acordo com a Ocorrência de peso ao Nascimento (2500 g em Gestação anterior).

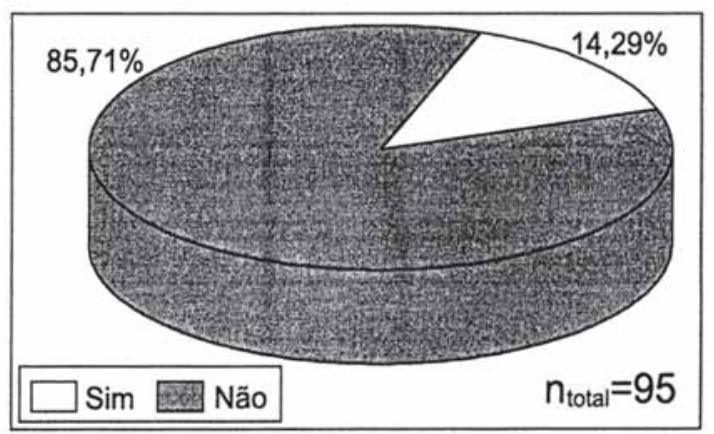

Tabela 2 - Distribuição das gestantes segundo fator de risco Anemia versus Estado Nutricional

\begin{tabular}{c|r|c|c|c|c}
\hline \multirow{2}{*}{$\begin{array}{c}\text { Estado } \\
\text { Nutricional }\end{array}$} & \multicolumn{4}{|c|}{ Anemia } & \multirow{2}{*}{ Total } \\
\cline { 2 - 5 } & \multicolumn{2}{|c|}{ Sim } & \multicolumn{2}{c}{ Não } & \\
\cline { 2 - 5 } & \multicolumn{1}{|c|}{ n } & $\%$ & $\mathrm{n}$ & $\%$ & \\
A & 11 & 55,00 & 15 & 28,30 & 26 \\
B & 7 & 35,00 & 14 & 26,42 & 21 \\
C & 2 & 10,00 & 24 & 45,28 & 26 \\
\hline Total & 20 & 100,00 & 53 & 100,00 & 73 \\
\hline
\end{tabular}

Teste do Qui-quadrado

$\mathrm{AxB} \rightarrow \mathrm{x}^{2}=0,40$

Teste Exato de Fisher

$\mathrm{BxC} \rightarrow \mathrm{p}=4,93$

$\mathrm{AxC} \rightarrow \mathrm{x}^{2}=8,31^{*}$

$\mathrm{x}^{2}$ Crítico $=3,84$

Tabela 3 - Distribuição das gestantes segundo Número de gestações versus Estado Nutricional

\begin{tabular}{c|r|c|c|c|c}
\hline \multirow{2}{*}{$\begin{array}{c}\text { Estado } \\
\text { Nutricional }\end{array}$} & \multicolumn{4}{|c|}{ Número de gestações } & \multirow{2}{*}{ Total } \\
\cline { 2 - 5 } & $<2$ & gestações & \multicolumn{2}{c}{$>2$ gestações } & \\
\cline { 2 - 5 } & n & $\%$ & $\mathrm{n}$ & $\%$ & \\
$\mathrm{~A}$ & 22 & 48,89 & 13 & 26,00 & 35 \\
$\mathrm{~B}$ & 16 & 35,56 & 13 & 26,00 & 29 \\
$\mathrm{C}$ & 7 & 15,56 & 24 & 48,00 & 31 \\
\hline Total & 45 & 100,00 & 50 & 100,00 & 95 \\
\hline \multicolumn{5}{c}{ Teste do Qui-quadrado } \\
AxB $\rightarrow \mathrm{x}^{2}=0,39$ & AxC $\rightarrow \mathrm{x}^{2}=10,83^{*}$ \\
$\mathrm{BxC} \rightarrow \mathrm{x}^{2}=6,73^{*}$ & $\mathrm{x}^{2}$ Critico $=3,84$
\end{tabular}

Figura 6 - Distribuição das Gestantes Segundo Número de Gestações

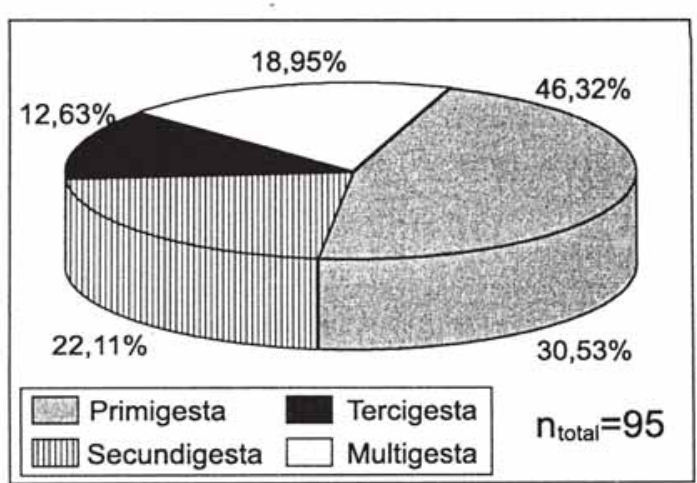

Tabela 4 - Distribuição das gestantes segundo fator de risco Infcção Urinária versus Estado Nutricional

\begin{tabular}{c|r|c|c|c|c}
\hline Estado & \multicolumn{4}{|c|}{ Infecção Urinária } & \multirow{2}{*}{ Total } \\
\cline { 2 - 5 } Nutricional & \multicolumn{3}{|c}{ Sim } & \multicolumn{1}{c}{ Não } & \\
\hline & $\mathrm{n}$ & $\%$ & $\mathrm{n}$ & $\%$ & \\
$\mathrm{~A}$ & 15 & 46,88 & 20 & 31,75 & 35 \\
$\mathrm{~B}$ & 8 & 25,00 & 21 & 33,33 & 29 \\
$\mathrm{C}$ & 9 & 28,13 & 22 & 34,92 & 31 \\
\hline Total & 32 & 100,00 & 63 & 100,00 & 95 \\
\hline \multicolumn{5}{|c}{ Teste do Qui-quadrado } \\
$\mathrm{AxB} \rightarrow \mathrm{x}^{2}=1,61$ & AxC $\rightarrow \mathrm{x}^{2}=1,36$ \\
$\mathrm{BxC} \rightarrow \mathrm{x}^{2}=0,02 \quad \mathrm{x}^{2}$ Critico $=3,84$
\end{tabular}


Tabela 5 - Distribuição das gestantes segundo ntervalo interpartal versus Estado Nutricional

\begin{tabular}{|c|c|c|c|c|c|}
\hline Estado & & ntervalo & terp & & TTat \\
\hline Nutricional & & anos & & anos & lotal \\
\hline & $\mathrm{n}$ & $\%$ & $\mathrm{n}$ & $\%$ & \\
\hline $\begin{array}{l}\text { A } \\
\text { B } \\
\text { C }\end{array}$ & $\begin{array}{l}5 \\
5 \\
9\end{array}$ & $\begin{array}{l}26,32 \\
26,32 \\
47,37\end{array}$ & $\begin{array}{r}8 \\
10 \\
11\end{array}$ & $\begin{array}{l}27,59 \\
34,48 \\
37,93\end{array}$ & $\begin{array}{l}13 \\
15 \\
20\end{array}$ \\
\hline Total & 19 & 100,00 & 29 & 100,00 & 48 \\
\hline $\begin{array}{l}\text { Teste do Qu } \\
\text { Teste Exato }\end{array}$ & & & $\begin{array}{l}\mathrm{AxB} \\
3 \times C \\
\mathrm{AxC} \\
{ }^{2} \mathrm{C}\end{array}$ & $\begin{array}{l}p=0,0 \\
x^{2}=0, \\
x^{2}=0, \\
\text { ico }=3,8\end{array}$ & \\
\hline
\end{tabular}

Outro fator de risco por nós avaliado, diz respeito ao intervalo interpartal. Em nosso estudo, não verificamos diferença estatisticamente significante do intervalo interpartal e a condição nutricional da gestante avaliada (Tabela 5). Para se efetivar o depósito de energia durante a gestação é necessário que o incremento alimentar realmente tenha ocorrido e, para a mãe que amamentou durante os 4 a 6 meses recomendados, estima-se em pelo menos 12 meses o tempo necessário para que esta reponha suas reservas, exis- tindo portanto, associação entre o baixo peso ao nascimento e intervalo interpartal (SOUZA QUEIROZ; PEREIRA \& NÓBREGA, 1998). Este fato, possivelmente, se deve ao curto período de amamentação desenvolvido pelas mães não permitndo o consumo adequado da gordura de depósito com a lactação; entretanto, estudos posteriores com casuisticas maiores são necessários para elucidar tal hipótese.

A monitorização do ganho de peso durante a gestação e o conhecimento dos inúmeros fatores de risco envolvidos na maiores: prevalência de baixo peso ao nascimento (prematuridade e desnutrição intra-uterina), são fundamentais para a efetiva prevenção destes agravos responsáveis por elevadas cifras dá mortalidade infantil (lembramos que hoje, as afecções perinatais são a nossa principal causa) e efeitos a curto e longo prazo na vitimização de nossas crianças já na vida intrauterina, con graves seqüelas envolvendo o crescimento/desenvolvimento. Medidas preventivas simples e facilmente exequíveis como a utilização da curva de ROSSO durante o exame pré-natal poderiam colaborar de forma significativa na redução do baixo peso ao nascimento, norteando medidas adequadas de orientação para as gestantes.

\begin{abstract}
The lack of monitoring ofthenutritional condition of pregnamtwomen during theprenatal exammat $\wedge$ lon has made it difficult for interventions aimir $g$ at the prevention of low weight at birth (BP). 45 pregnmt women of low socio-economic levei registered $m$ the posts of the FOUNDATION for ASSISTANCE to INFANCY (FAISA - Santo André, São Paulo) were evaluated, for accomplishment of prenatal exammation, using Rosso's Graph for its nutritional classification. The achieved percentage of pregnantwomenwi^th low weightwas $36,8 \%$. Mo ${ }^{\wedge}$ thers wi^thmore thamtwo ges $\wedge$ tations and $\mathrm{wi}^{\wedge} \wedge$ th less than two presented greater occurrence of overweight obesi^ty amd low weight, respectively. Higher percentage of amemia in the pregmant women with low weight was verified. Therefore, it is evident that this instrument should be implemented as routine in $\wedge$ the prenatal examinabon.
\end{abstract}

Key-wrds: pregnamt woman; low weight at birth infants; prenatal.

\section{REFERÊNCIAS BIBLIOGRÁFICAS}

BRASIL, Mmistério da Saúde - instituto Nacional de Alimentação e Nutrição - Pesquisa Nacional sobre Demografia e Saúde (PNDS), 1996,

BRASIL, Ministéno da Saúde. Secretaria Nacional de Programas Especiais de Saúde. Divisão Nacional - SNPES/DINSAMI- Assisfencia Pré-Natal. Brasília, Centro de Documentação do Ministério da Saúde, 1998 (Normas e M anuais Técnicos, 36).

CANTIN, A.; CRYSTAL, L. R. G. Oxid mts, amtioxidants and the $\mathrm{pa}^{\wedge}$ thogenesis of enfisema. Eur . Respir Dis., 66 (Suppl 139): 7-17, 1985,
GODFREY, K.; ROBINSON, S. Maternal nutrition, placental growth amd fetal programming. Proc. Nutr Soc.,57: 105-111, 1998.

KELLY, A.; KEVANY, J.; ONIS, M.; SHAH, P. M. A who collaborative study of maternal anthropometry and pregnancy outcomes. Int.: Ginecol \& Obstetr., s3: 219-233, 1996.

LUCENA, L.; LIMA, R. T; MARINO, W T O baixo peso ao nascer ainda é um problema de Saúde Pública no Brasil. Rev. Paul. Pediatria, 16(1): 15-25, 1998.

MARIOTON 1, G. G B.; BARROS FILHO, AA. A gravidez na adolescência é fator de nsco para O baixo peso ao nascer. J. Pediatr., 74(2): 107118, 1998. 
PEREIRA, R.T Avaliação da condição nutricional das gestantes do Estado de São Paulo, por antropometria, utilizando o Grafico de Rosso. São Paulo, 1993. [Dissertação de Mestrado em Nutrição da UNIFESP/EPM].

PINTO, F. S. Medida da espessura da prega cutânea do recém-nascido e sua relação com a condição nutricional materna. Botucatu, 1996. [Tese de Mestrado - UNESP].

PUFFER, R. R; SERRANO, C. V. Patterns of mortality in childhood. Washington, Pan American Health Organization, 1973. (Scientific Publication, 262).

PUFFER, R. R; SERRANO, C. V. Características del peso la nacer. Washington, Organización Panamericana de la Salud, 1987. (Publicación científica, 504).

ROCHA, J. A. Baixo Peso, Peso Insuficiente e Peso Adequado ao Nascer, em 5.940 nascidos vivos na cidade do Recife. J. Ped., 67 (9/10): 297-304, 1991.

ROSSO, P. A new chart to monitor weight gain during pregnancy. Am. J. Clin. Nutr., 41: 644652, 1985.
SIEGEL, S. Estatística no parametrica. México, Ed. Trillas, 1975. p. 346.

SINISTERRA-RODRIGUEZ, O. T. Anemia e desnutrição maternas e sua relação com o peso ao nascei Rev. Saúde Pública, 25 (3): 193-197, 1991.

SOUZA QUEIROZ, S. S.; PEREIRA, R. T.; NÓBREGA, F. J. Fatores de risco. In: NÓBREGA, F. J. Distúrbios nutrição. Rio de Janeiro, Revinter, 1998. p. 109-116.

TADDEI, J. A .; JACOMO, A. J.; ALBUQUERQUE, Z. R; AQUINO, M. H. B.; MATHIAS, S. P.; TORRES, 1. H. B.; SEGOVIA, I.; SRIEDES, R. M.; MACHADO, C. S.; SANTOS, P. S. M.; CAMPELLO, L. The weight for height chart on the prognosis of loa birth weight: a multicentric study. Rev. Bras. Ginecol. Obstet., 2: 78-82, 1991.

VITALLE, M. S. S. Recém-nascido de mãe adolescente de baixo nível sócio-econômico (antropometriac outras variáveis). São Paulo, 1994. [Tese de Mestrado - EPM/UNIFESP]. 